\title{
Risk Factors of Epistaxis in Primary School Children in Dakahlia Governorate, Egypt
}

\author{
Huda M. EL-Baz*1, Khalid A. Mohammed ${ }^{2}$, Hanaa S. Said ${ }^{3}$
}

Department of ${ }^{1}$ Family Medicine in Sanafa Family Health Center - Mit-Ghamr City Dakahlia Governorate, Egypt

Departments of ${ }^{2}$ Ear, Nose and Throat and ${ }^{3}$ Family Medicine, Faculty of Medicine, Zagazig University, Egypt

*Corresponding author: Huda Mohamed EL-Baz, Mobile: (+20) 01115163402, Email: dedo.m7med567@gmail.com

\begin{abstract}
Background: Epistaxis is a common pediatric complaint, so we study its risk factors trying to control and decrease it among primary school children.

Objectives: To determine frequency of epistaxis and to assess the risk factors of epistaxis in primary school children (8-12 years old).

Patients and Methods: A cross-sectional study that was conducted on 312 children. Sample was collected by systematic random technique from the children attending to Family Health Center, Sanafa, Mit-Ghamr city, Dakahlia Governorate, Egypt. Participants' data regarding socio-demographic characteristics, and risk factors were collected via predesigned questionnaire.

Results: The study revealed that prevalence of epistaxis among the studied group was $32.4 \%$ and the most common risk factors among the studied group were smoking, head trauma, factory gases, chronic cough and upper respiratory tract infection (URTI) $(56.7 \%, 45.8 \%, 35.6 \%, 32.7 \% \& 30.1 \%$ respectively).

Conclusion: Recurrent epistaxis can be troublesome and alarming for parents and children. so special attention must be given for it.
\end{abstract}

Keywords: Epistaxis, Risk factors, Primary school children.

\section{INTRODUCTION}

Hemorrhages from the nasal cavity are called epistaxis or nose bleeds. Epistaxis is not a disease; it is a symptom. It is a highly common ear, nose and throat emergency. Yet its exact prevalence is not known, and studies report a wide range about $60 \%$ cases in the population, with only $6 \%$ of those seeing a physician. Epistaxis is rarely life threatening but may cause significant concern, especially among parents of young children ${ }^{(\mathbf{1})}$.

Children may be more prone to nose bleeds due to the extensive vascular supply and increased frequency of upper respiratory tract infection. The natural history of the problem is one of intermittent, recurrent and usually minor nose bleeds that mostly nonspontaneous, but which can cause alarm $^{(2)}$.

Epistaxis is affecting up to $56 \%$ aged 6-10 years and around $9 \%$ of teenaged population ${ }^{(3)}$.

Severe and recurrent forms of epistaxis are considered a major trouble to the parents and treating doctors. Causes of recurrent epistaxis look different between adult and children regarding frequency and distribution of such causes ${ }^{(4)}$.

The etiology of epistaxis can be divided into local or systemic causes, although this distinction is difficult to make and the term "Idiopathic Epistaxis" represents about $80-90 \%$ of the cases. The etiological profile of epistaxis has been reported to vary with age and anatomical location. Traumatic epistaxis is more common in younger individuals and is most often due to digital trauma, facial injury, or a foreign body in the nasal cavity. Nontraumatic epistaxis is rare in children and may be due to systemic diseases and environmental factors (temperature, humidity, altitude). Epistaxis that occurs in children younger than 10 years usually is mild and originates in the anterior nose ${ }^{(5)}$.

\section{Research question:}

What about the frequency of epistaxis among primary school children (between 6 and 12 years old)? And what are its risk factors?

The aim of the present study was to determine frequency of epistaxis and to assess its risk factors in primary school children (8-12 years old).

\section{PATIENTS AND METHODS}

A cross-sectional study that was conducted on 312 children. As the total attendance rate of the target aged children at Sanafa, Mit-Ghamr Family Health Center was 1740 in six months and the prevalence of epistaxis was $56 \%{ }^{(3)}$, the power of the study was $80 \%$ and CI was $95 \%$ by open epi program version 3 .

Sample was collected by systematic random technique from the children attending the previously mentioned health unit asking for any medical service. 
Researchers gave questionnaire to every fourth child coming to the unit to reach 20 children per day and data were collected day after day, 3 times per week.

A predesigned questionnaire fulfilled by the researchers by personal interview with the child's guardian (mother or father) attending with the child. The questionnaire consisted of socio-demographic assessment questions, ${ }^{(6)}$ medical characteristics and risk factors for epistaxis of the studied children ${ }^{(7)}$.

\section{Administrative and ethical approval:}

Official permission was obtained from The Scientific Ethical Committee of the Family Medicine Department, Faculty of Medicine, Zagazig University [(IRB) \#;4611/16-5-2018].

Official approval letter was taken to the manager of the Family Health Center to obtain the information from children attending to the center.

All subjects in this study were interviewed after taking their consent (child's parents) and assuring confidentiality of their information. This work has been carried out in accordance with The Code of Ethics of the World Medical Association (Declaration of Helsinki) for studies involving humans.

\section{Statistical analysis}

The collected data were computerized and statistically analyzed using SPSS program (Statistical Package for Social Science) version 25.0. Qualitative data were represented as frequencies and relative percentages. Chi square test was used to calculate difference between qualitative variables.

\section{RESULTS}

Regarding children ages and gender, $60.9 \%$ were from 10 to 12 years old and $55.8 \%$ were males. About two thirds of mothers and fathers were highly educated. Also $91.3 \%$ of fathers were working and $52.2 \%$ of mothers were housewives. $52.9 \%$ were of high social class. The prevalence of epistaxis among the studied group was $32.4 \%$ (Table $1 \&$ figure 1 ).
Table (1): Demographic data of the studied group

\begin{tabular}{|c|c|c|c|}
\hline & Variable & \multicolumn{2}{|c|}{$(n=312)$} \\
\hline $\begin{array}{l}\text { Father age: } \\
\text { (years) }\end{array}$ & $\begin{array}{c}\text { Mean } \pm \text { SD } \\
\text { Range }\end{array}$ & \multicolumn{2}{|c|}{$\begin{array}{c}41.92 \pm 6.04 \\
30-56\end{array}$} \\
\hline $\begin{array}{l}\text { Mother age: } \\
\text { (years) }\end{array}$ & $\begin{array}{c}\text { Mean } \pm \text { SD } \\
\text { Range }\end{array}$ & \multicolumn{2}{|c|}{$\begin{array}{c}38.04 \pm 5.92 \\
25-52\end{array}$} \\
\hline & Variable & No & $\%$ \\
\hline Age group: & $\begin{array}{c}7-9 \\
10-12\end{array}$ & $\begin{array}{l}122 \\
190\end{array}$ & $\begin{array}{l}39.1 \\
60.9\end{array}$ \\
\hline Sex: & $\begin{array}{c}\text { Male } \\
\text { Female }\end{array}$ & $\begin{array}{l}174 \\
138 \\
\end{array}$ & $\begin{array}{l}55.8 \\
44.2 \\
\end{array}$ \\
\hline $\begin{array}{l}\text { Father } \\
\text { education: }\end{array}$ & $\begin{array}{c}\text { Illiterate } \\
\text { Read \& write } \\
\text { secondary school } \\
\text { High education }\end{array}$ & $\begin{array}{c}23 \\
1 \\
83 \\
205 \\
\end{array}$ & $\begin{array}{c}7.4 \\
0.3 \\
26.6 \\
65.7 \\
\end{array}$ \\
\hline $\begin{array}{l}\text { Mother } \\
\text { education: }\end{array}$ & $\begin{array}{c}\text { Illiterate } \\
\text { Read \& write } \\
\text { primary school } \\
\text { secondary school } \\
\text { High education }\end{array}$ & $\begin{array}{c}23 \\
1 \\
1 \\
94 \\
193\end{array}$ & $\begin{array}{c}7.4 \\
0.3 \\
0.3 \\
30.1 \\
61.9\end{array}$ \\
\hline $\begin{array}{l}\text { Father working } \\
\text { status: }\end{array}$ & $\begin{array}{l}\text { Yes } \\
\text { No }\end{array}$ & $\begin{array}{c}285 \\
27\end{array}$ & $\begin{array}{c}91.3 \\
8.7\end{array}$ \\
\hline $\begin{array}{l}\text { Mother working } \\
\text { status: }\end{array}$ & $\begin{array}{l}\text { Yes } \\
\text { No }\end{array}$ & $\begin{array}{l}149 \\
163 \\
\end{array}$ & $\begin{array}{l}47.8 \\
52.2 \\
\end{array}$ \\
\hline Social class: & $\begin{array}{c}\text { Low class } \\
\text { Moderate } \\
\text { High }\end{array}$ & $\begin{array}{c}73 \\
74 \\
165 \\
\end{array}$ & $\begin{array}{l}23.4 \\
23.7 \\
52.9 \\
\end{array}$ \\
\hline
\end{tabular}

\section{Epistaxis}

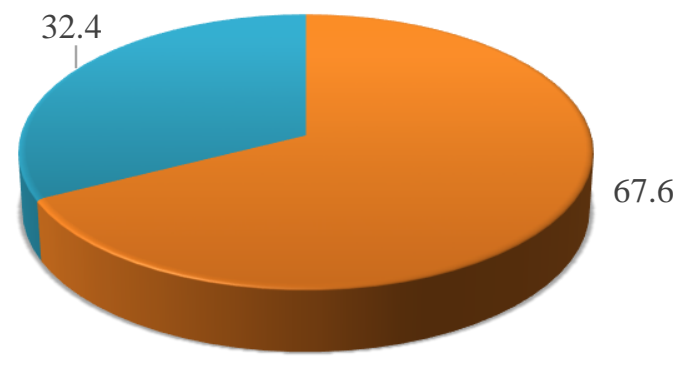

$\square$ No

$\square$ Yes

Figure (1): Frequency of epistaxis among the studied group

The most common risk factors among the studied group were smoking, head trauma, factory gases, chronic cough and URTI $(56.7 \%, 45.8 \%, 35.6 \%, 32.7 \%$ \& $30.1 \%$ respectively) (Table 2 ). 
Table (2): Risk factor for epistaxis among the studied group

\begin{tabular}{|c|c|c|c|}
\hline \multirow{2}{*}{\multicolumn{2}{|c|}{ Variable }} & \multicolumn{2}{|c|}{$(n=312)$} \\
\hline & & \multirow{2}{*}{$\begin{array}{l}\text { No } \\
169\end{array}$} & \multirow{2}{*}{$\frac{\%}{54.2}$} \\
\hline Head trauma: & No & & \\
\hline & Yes & 143 & 45.8 \\
\hline \multirow[t]{2}{*}{ Anti-histaminic or cortisol: } & No & 259 & 83 \\
\hline & Yes & 53 & 17 \\
\hline \multirow[t]{2}{*}{ Nasal spray: } & No & 288 & 92.3 \\
\hline & Yes & 24 & 7.7 \\
\hline \multirow[t]{2}{*}{ Septal deformity: } & No & 274 & 87.8 \\
\hline & Yes & 38 & 12.2 \\
\hline \multirow[t]{2}{*}{ Allergic rhinitis: } & No & 256 & 82.1 \\
\hline & Yes & 56 & 17.9 \\
\hline \multirow[t]{2}{*}{ Sinusitis: } & No & 273 & 87.5 \\
\hline & Yes & 39 & 12.5 \\
\hline \multirow[t]{2}{*}{ Upper respiratory tract infection: } & No & 218 & 69.9 \\
\hline & Yes & 94 & 30.1 \\
\hline \multirow[t]{2}{*}{ Smoking: } & No & 135 & 43.3 \\
\hline & Yes & 177 & 56.7 \\
\hline \multirow[t]{2}{*}{ Factory gases: } & No & 201 & 64.4 \\
\hline & Yes & 111 & 35.6 \\
\hline \multirow[t]{2}{*}{ Chronic cough: } & No & 201 & 67.3 \\
\hline & Yes & 102 & 32.7 \\
\hline \multirow[t]{2}{*}{ Any nasal or body tumors: } & No & 301 & 96.5 \\
\hline & Yes & 11 & 3.5 \\
\hline \multirow[t]{2}{*}{ Hemophilia: } & No & 289 & 92.6 \\
\hline & Yes & 23 & 7.4 \\
\hline \multirow[t]{2}{*}{ Decreased platelets count: } & No & 299 & 95.8 \\
\hline & Yes & 13 & 4.2 \\
\hline \multirow[t]{2}{*}{ Liver disorders: } & No & 291 & 93.3 \\
\hline & Yes & 21 & 6.7 \\
\hline \multirow[t]{2}{*}{ Anticoagulant disorders: } & No & 304 & 97.4 \\
\hline & Yes & 8 & 2.6 \\
\hline \multirow[t]{2}{*}{ Vascular disease: } & No & 270 & 86.5 \\
\hline & Yes & 42 & 13.5 \\
\hline \multirow[t]{2}{*}{ Migraine: } & No & 283 & 90.7 \\
\hline & Yes & 29 & 9.3 \\
\hline \multirow[t]{2}{*}{ HPT: } & No & 295 & 94.6 \\
\hline & Yes & 17 & 5.4 \\
\hline \multirow[t]{2}{*}{ Frequent aero plane travelling: } & No & 301 & 96.5 \\
\hline & Yes & 11 & 3.5 \\
\hline
\end{tabular}

Table (3) showed that 51.5\% of the studied group had epistaxis from 1 to 4 years ago and 69.2 had it 1-2 times per month. Regarding seasonal variation, $67.3 \%$ reported increase in summer. In $61.4 \%$ of the cases it was associated with fatigue and lethargy, $57.4 \%$ were associated with scratching inside nose, $74.3 \%$ were idiopathic, $29.7 \%$ were after foreign body (FB) entrance and $6.9 \%$ were associated with travelling with plane. Finally, $32.7 \%$ had family history and $28.7 \%$ had $+\mathrm{ve}$ consanguinity. 
Table (3): History of epistasis among the affected cases

\begin{tabular}{|c|c|c|c|}
\hline \multirow{2}{*}{\multicolumn{2}{|c|}{ Variable }} & \multicolumn{2}{|c|}{$(n=101)$} \\
\hline & & No & $\%$ \\
\hline Onset of the $1^{\text {st }}$ time: & $\begin{array}{l}<1 y \\
1-4 y \\
5-9 y\end{array}$ & $\begin{array}{l}28 \\
52 \\
21 \\
\end{array}$ & $\begin{array}{l}27.7 \\
51.5 \\
20.8 \\
\end{array}$ \\
\hline Rate: & $\begin{array}{l}\text { 1-2 times } / \mathrm{m} \\
3-4 \text { times } / \mathrm{m} \\
>4 \text { times } / \mathrm{m}\end{array}$ & $\begin{array}{c}70 \\
26 \\
5\end{array}$ & $\begin{array}{c}69.2 \\
25.8 \\
5\end{array}$ \\
\hline Amount: & $\begin{array}{c}\text { Mild } \\
\text { Moderate } \\
\text { Sever }\end{array}$ & $\begin{array}{l}31 \\
50 \\
20 \\
\end{array}$ & $\begin{array}{l}30.7 \\
49.5 \\
19.8 \\
\end{array}$ \\
\hline Seasonal affection: & $\begin{array}{c}\text { Autumn } \\
\text { Spring } \\
\text { Summer } \\
\text { Winter } \\
\text { All }\end{array}$ & $\begin{array}{c}1 \\
13 \\
68 \\
18 \\
1\end{array}$ & $\begin{array}{c}1 \\
12.9 \\
67.3 \\
17.8 \\
1\end{array}$ \\
\hline Associated with fatigue \& lethargy: & $\begin{array}{l}\text { No } \\
\text { Yes }\end{array}$ & $\begin{array}{l}39 \\
62\end{array}$ & $\begin{array}{l}38.6 \\
61.4\end{array}$ \\
\hline After nasal scratch: & $\begin{array}{l}\text { No } \\
\text { Yes }\end{array}$ & $\begin{array}{l}43 \\
58\end{array}$ & $\begin{array}{l}42.6 \\
57.4\end{array}$ \\
\hline Idiopathic: & $\begin{array}{l}\text { No } \\
\text { Yes }\end{array}$ & $\begin{array}{l}26 \\
75\end{array}$ & $\begin{array}{l}25.7 \\
74.3\end{array}$ \\
\hline Due to FB entrance: & $\begin{array}{l}\text { No } \\
\text { Yes }\end{array}$ & $\begin{array}{l}71 \\
30\end{array}$ & $\begin{array}{l}70.3 \\
29.7\end{array}$ \\
\hline With plane travelling: & $\begin{array}{l}\text { No } \\
\text { Yes }\end{array}$ & $\begin{array}{c}94 \\
7\end{array}$ & $\begin{array}{c}93.1 \\
6.9\end{array}$ \\
\hline Family history: & $\begin{array}{l}\text { No } \\
\text { Yes }\end{array}$ & $\begin{array}{l}68 \\
33 \\
\end{array}$ & $\begin{array}{l}67.3 \\
32.7 \\
\end{array}$ \\
\hline Consanguinity: & $\begin{array}{l}\text { No } \\
\text { Yes }\end{array}$ & $\begin{array}{l}72 \\
29\end{array}$ & $\begin{array}{l}71.3 \\
28.7\end{array}$ \\
\hline
\end{tabular}

Figure (3) showed that severity of epistaxis among the affected cases was divided into sever $(30,7 \%)$, moderate $(49,5 \%)$ and mild $(19,8 \%)$.

\section{Amount}

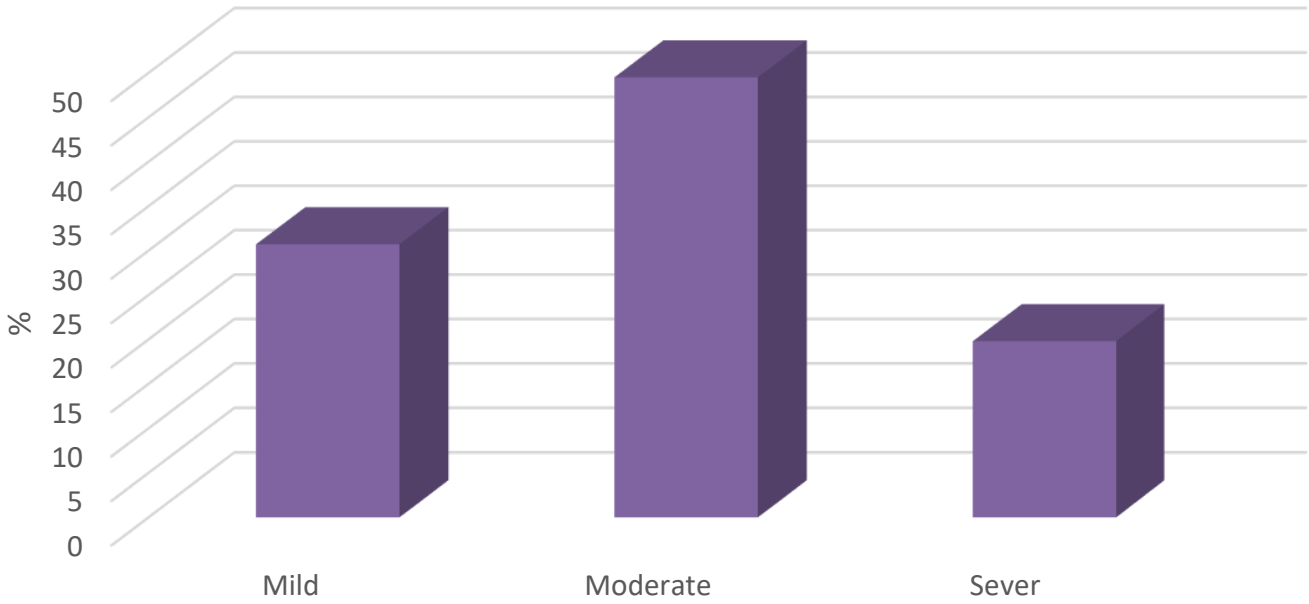

Figure (3): Severity of epistaxis among the affected cases 
https://ejhm.journals.ekb.eg/

The cases whose age was between 7 and 9 years were about 2 times more exposed to have epistaxis (Table 4).

Table (4): Comparison between cases had epistaxis and cases hadn't in demographic data

\begin{tabular}{|c|c|c|c|c|c|c|c|c|c|}
\hline \multirow{2}{*}{\multicolumn{2}{|c|}{ Variable }} & \multirow{3}{*}{$\begin{array}{c}\text { Total } \\
122 \\
190\end{array}$} & \multicolumn{2}{|c|}{ Hadn't epistaxis (n=211) } & \multicolumn{2}{|c|}{ Had epistaxis $(\mathrm{n}=101)$} & \multirow{2}{*}{$\chi^{2}$} & \multirow[b]{2}{*}{$\mathbf{P}$} & \multirow{2}{*}{$\begin{array}{c}\text { OR } \\
\text { (CI 95\%) }\end{array}$} \\
\hline & & & No & $\%$ & No & $\%$ & & & \\
\hline $\begin{array}{c}\text { Age group: } \\
\text { (years) }\end{array}$ & $\begin{array}{c}7-9 \\
10-12\end{array}$ & & $\begin{array}{c}71 \\
140\end{array}$ & $\begin{array}{l}58.2 \\
73.7\end{array}$ & $\begin{array}{l}51 \\
50\end{array}$ & $\begin{array}{l}41.8 \\
26.3\end{array}$ & 8.14 & $\begin{array}{c}0.004 \\
* *\end{array}$ & $\begin{array}{c}2.01 \\
(1.24-3.26)\end{array}$ \\
\hline Sex & $\begin{array}{c}\text { Female } \\
\text { Male }\end{array}$ & $\begin{array}{l}138 \\
174 \\
\end{array}$ & $\begin{array}{c}98 \\
113 \\
\end{array}$ & $\begin{array}{c}71 \\
64.9 \\
\end{array}$ & $\begin{array}{l}40 \\
61 \\
\end{array}$ & $\begin{array}{c}29 \\
35.1 \\
\end{array}$ & 1.30 & $\begin{array}{c}0.26 \\
\text { NS }\end{array}$ & $\begin{array}{c}1.32 \\
(0.82-2.14)\end{array}$ \\
\hline Social class & $\begin{array}{c}\text { Low } \\
\text { Moderate } \\
\text { High }\end{array}$ & $\begin{array}{c}74 \\
165 \\
73 \\
\end{array}$ & $\begin{array}{c}51 \\
111 \\
49 \\
\end{array}$ & $\begin{array}{l}68.9 \\
67.3 \\
67.1 \\
\end{array}$ & $\begin{array}{l}23 \\
54 \\
24 \\
\end{array}$ & $\begin{array}{l}31.1 \\
32.7 \\
32.9 \\
\end{array}$ & 0.07 & $\begin{array}{c}0.96 \\
\text { NS }\end{array}$ & ---- \\
\hline
\end{tabular}

$\chi^{2}$ :Chi square test.

NS: Non significant $(\mathrm{P}<0.05) \quad * *$ : Highly significant $(\mathrm{P}<0.01)$.

OR: Odds ratio

Comparison between cases had epistaxis and cases hadn't in risk factors showed that the cases had head trauma were about 8 times more exposed to have epistaxis. Also, cases used nasal spray were 5 times more exposed to have epistaxis and cases had hemophilia were about 7 times more exposed to have epistaxis. Finally, vascular disease increased risk of epistaxis 145 times (Table 5).

Table (5): Comparison between cases had epistaxis and cases hadn't in Risk factor

\begin{tabular}{|c|c|c|c|c|c|c|c|c|c|}
\hline \multirow{2}{*}{\multicolumn{2}{|c|}{ Variable }} & \multirow{3}{*}{$\begin{array}{c}\text { Total } \\
169 \\
143 \\
\end{array}$} & \multicolumn{2}{|c|}{ Hadn't epistaxis $(n=211)$} & \multicolumn{2}{|c|}{ Had epistaxis (n=101) } & \multirow[t]{2}{*}{$\chi^{2}$} & \multirow[t]{2}{*}{$\mathbf{P}$} & \multirow{2}{*}{$\begin{array}{c}\text { OR } \\
\text { (CI 95\%) }\end{array}$} \\
\hline & & & No & $\%$ & No & $\%$ & & & \\
\hline Head trauma: & $\begin{array}{l}\text { No } \\
\text { Yes }\end{array}$ & & $\begin{array}{c}147 \\
64\end{array}$ & $\begin{array}{c}87 \\
44.8\end{array}$ & $\begin{array}{l}22 \\
79\end{array}$ & $\begin{array}{c}13 \\
55.2\end{array}$ & 63.09 & $\begin{array}{c}<0.001 \\
* *\end{array}$ & $\begin{array}{c}8.25 \\
(4.72-14.39)\end{array}$ \\
\hline $\begin{array}{l}\text { Anti-histaminic or } \\
\text { cortisol: }\end{array}$ & $\begin{array}{l}\text { No } \\
\text { Yes }\end{array}$ & $\begin{array}{c}259 \\
53\end{array}$ & $\begin{array}{c}180 \\
31\end{array}$ & $\begin{array}{l}69.5 \\
58.5\end{array}$ & $\begin{array}{l}79 \\
22\end{array}$ & $\begin{array}{l}30.5 \\
41.5\end{array}$ & 2.44 & $\begin{array}{l}0.12 \\
\mathrm{NS}\end{array}$ & $\begin{array}{c}1.62 \\
(0.88-2.97)\end{array}$ \\
\hline Nasal spray: & $\begin{array}{l}\text { No } \\
\text { Yes }\end{array}$ & $\begin{array}{l}288 \\
24\end{array}$ & $\begin{array}{c}203 \\
8\end{array}$ & $\begin{array}{l}70.5 \\
33.3\end{array}$ & $\begin{array}{l}85 \\
16\end{array}$ & $\begin{array}{l}29.5 \\
66.7\end{array}$ & 13.97 & $\begin{array}{c}<0.001 \\
* *\end{array}$ & $\begin{array}{c}4.78 \\
(1.97-11.58)\end{array}$ \\
\hline Septal deformity: & $\begin{array}{l}\text { No } \\
\text { Yes } \\
\end{array}$ & $\begin{array}{l}274 \\
38 \\
\end{array}$ & $\begin{array}{c}186 \\
25 \\
\end{array}$ & $\begin{array}{l}67.9 \\
65.8 \\
\end{array}$ & $\begin{array}{l}88 \\
13 \\
\end{array}$ & $\begin{array}{l}32.1 \\
34.2 \\
\end{array}$ & 0.07 & $\begin{array}{c}0.80 \\
\mathrm{NS}\end{array}$ & $\begin{array}{c}1.10 \\
(0.54-2.25) \\
\end{array}$ \\
\hline Allergic rhinitis: & $\begin{array}{l}\text { No } \\
\text { Yes }\end{array}$ & $\begin{array}{c}256 \\
56\end{array}$ & $\begin{array}{c}177 \\
34\end{array}$ & $\begin{array}{l}69.1 \\
60.7 \\
\end{array}$ & $\begin{array}{l}79 \\
22 \\
\end{array}$ & $\begin{array}{l}30.9 \\
39.3 \\
\end{array}$ & 1.49 & $\begin{array}{l}0.22 \\
\mathrm{NS}\end{array}$ & $\begin{array}{c}1.45 \\
(0.80-2.64)\end{array}$ \\
\hline Sinusitis: & $\begin{array}{l}\text { No } \\
\text { Yes }\end{array}$ & $\begin{array}{l}273 \\
39\end{array}$ & $\begin{array}{c}188 \\
23\end{array}$ & $\begin{array}{c}68.9 \\
59\end{array}$ & $\begin{array}{l}85 \\
16\end{array}$ & $\begin{array}{c}31.1 \\
41\end{array}$ & 1.53 & $\begin{array}{l}0.22 \\
\mathrm{NS}\end{array}$ & $\begin{array}{c}1.54 \\
(0.77-3.06)\end{array}$ \\
\hline $\begin{array}{l}\text { Upper respiratory } \\
\text { tract infection: }\end{array}$ & $\begin{array}{l}\text { No } \\
\text { Yes }\end{array}$ & $\begin{array}{c}218 \\
94\end{array}$ & $\begin{array}{c}152 \\
59\end{array}$ & $\begin{array}{l}69.7 \\
62.8\end{array}$ & $\begin{array}{l}66 \\
35\end{array}$ & $\begin{array}{l}30.3 \\
37.2\end{array}$ & 1.45 & $\begin{array}{l}0.23 \\
\mathrm{NS}\end{array}$ & $\begin{array}{c}1.37 \\
(0.82-2.27)\end{array}$ \\
\hline Smoking: & $\begin{array}{l}\text { No } \\
\text { Yes }\end{array}$ & $\begin{array}{l}135 \\
177\end{array}$ & $\begin{array}{c}93 \\
118\end{array}$ & $\begin{array}{l}68.9 \\
66.7\end{array}$ & $\begin{array}{l}42 \\
59\end{array}$ & $\begin{array}{l}31.1 \\
33.3\end{array}$ & 0.17 & $\begin{array}{l}0.68 \\
\mathrm{NS}\end{array}$ & $\begin{array}{c}1.11 \\
(0.69-1.79)\end{array}$ \\
\hline Factory gases: & $\begin{array}{l}\text { No } \\
\text { Yes }\end{array}$ & $\begin{array}{l}201 \\
111\end{array}$ & $\begin{array}{c}135 \\
76\end{array}$ & $\begin{array}{l}67.2 \\
68.5\end{array}$ & $\begin{array}{l}66 \\
35\end{array}$ & $\begin{array}{l}32.8 \\
31.5\end{array}$ & 0.06 & $\begin{array}{l}0.81 \\
\mathrm{NS}\end{array}$ & $\begin{array}{c}0.94 \\
(0.57-1.55)\end{array}$ \\
\hline Chronic cough: & $\begin{array}{l}\text { No } \\
\text { Yes }\end{array}$ & $\begin{array}{l}201 \\
102\end{array}$ & $\begin{array}{c}137 \\
74\end{array}$ & $\begin{array}{l}65.2 \\
72.5\end{array}$ & $\begin{array}{l}73 \\
28\end{array}$ & $\begin{array}{l}34.8 \\
27.5\end{array}$ & 1.68 & $\begin{array}{l}0.20 \\
\mathrm{NS}\end{array}$ & $\begin{array}{c}0.71 \\
(0.42-1.19)\end{array}$ \\
\hline $\begin{array}{l}\text { Any nasal or body } \\
\text { tumors: }\end{array}$ & $\begin{array}{l}\text { No } \\
\text { Yes }\end{array}$ & $\begin{array}{c}301 \\
11\end{array}$ & $\begin{array}{c}202 \\
9\end{array}$ & $\begin{array}{l}67.1 \\
81.8 \\
\end{array}$ & $\begin{array}{c}99 \\
2\end{array}$ & $\begin{array}{l}32.9 \\
18.2 \\
\end{array}$ & 1.05 & $\begin{array}{l}0.31 \\
\text { NS }\end{array}$ & $\begin{array}{c}0.45 \\
(0.10-2.14)\end{array}$ \\
\hline Hemophilia: & $\begin{array}{l}\text { No } \\
\text { Yes }\end{array}$ & $\begin{array}{c}289 \\
23\end{array}$ & $\begin{array}{c}205 \\
6\end{array}$ & $\begin{array}{l}70.9 \\
26.1\end{array}$ & $\begin{array}{l}84 \\
17\end{array}$ & $\begin{array}{l}29.1 \\
73.9\end{array}$ & 19.57 & $\begin{array}{c}<0.001 \\
* *\end{array}$ & $\begin{array}{c}6.92 \\
(2.64-18.14)\end{array}$ \\
\hline $\begin{array}{l}\text { Decreased platelets } \\
\text { count: }\end{array}$ & $\begin{array}{l}\text { No } \\
\text { Yes }\end{array}$ & $\begin{array}{c}299 \\
13 \\
\end{array}$ & $\begin{array}{c}205 \\
6\end{array}$ & $\begin{array}{l}68.6 \\
46.2\end{array}$ & $\begin{array}{c}94 \\
7\end{array}$ & $\begin{array}{l}31.4 \\
53.8\end{array}$ & 2.86 & $\begin{array}{l}0.09 \\
\text { NS }\end{array}$ & $\begin{array}{c}2.54 \\
(0.83-7.78)\end{array}$ \\
\hline Liver disorders: & $\begin{array}{l}\text { No } \\
\text { Yes }\end{array}$ & $\begin{array}{c}291 \\
21\end{array}$ & $\begin{array}{c}193 \\
18\end{array}$ & $\begin{array}{l}66.3 \\
85.7\end{array}$ & $\begin{array}{c}98 \\
3\end{array}$ & $\begin{array}{l}33.7 \\
14.3 \\
\end{array}$ & 3.36 & $\begin{array}{l}0.07 \\
\mathrm{NS}\end{array}$ & $\begin{array}{c}0.33 \\
(0.09-1.14)\end{array}$ \\
\hline $\begin{array}{l}\text { Anticoagulant } \\
\text { disorders: }\end{array}$ & $\begin{array}{l}\text { No } \\
\text { Yes }\end{array}$ & $\begin{array}{c}304 \\
8\end{array}$ & $\begin{array}{c}208 \\
3\end{array}$ & $\begin{array}{l}68.4 \\
37.5\end{array}$ & $\begin{array}{c}96 \\
5\end{array}$ & $\begin{array}{l}31.6 \\
62.5\end{array}$ & 3.40 & $\begin{array}{l}0.07 \\
\mathrm{NS}\end{array}$ & $\begin{array}{c}3.61 \\
(0.85-15.42)\end{array}$ \\
\hline Vascular disease: & $\begin{array}{l}\text { No } \\
\text { Yes }\end{array}$ & $\begin{array}{c}270 \\
42\end{array}$ & $\begin{array}{c}210 \\
1\end{array}$ & $\begin{array}{c}77.8 \\
2.4\end{array}$ & $\begin{array}{l}60 \\
41\end{array}$ & $\begin{array}{l}22.2 \\
97.6\end{array}$ & 94.38 & $\begin{array}{c}<0.001 \\
* *\end{array}$ & $\begin{array}{c}143.5 \\
(19.33-1064)\end{array}$ \\
\hline Migraine: & $\begin{array}{l}\text { No } \\
\text { Yes }\end{array}$ & $\begin{array}{l}283 \\
29\end{array}$ & $\begin{array}{c}190 \\
21\end{array}$ & $\begin{array}{l}67.1 \\
72.4\end{array}$ & $\begin{array}{c}93 \\
8\end{array}$ & $\begin{array}{l}32.9 \\
27.6\end{array}$ & 0.33 & $\begin{array}{l}0.56 \\
\mathrm{NS}\end{array}$ & $\begin{array}{c}0.78 \\
(0.33-1.82)\end{array}$ \\
\hline HPT: & $\begin{array}{l}\text { No } \\
\text { Yes }\end{array}$ & $\begin{array}{c}295 \\
17 \\
\end{array}$ & $\begin{array}{c}198 \\
13 \\
\end{array}$ & $\begin{array}{l}67.1 \\
76.5 \\
\end{array}$ & $\begin{array}{c}97 \\
4\end{array}$ & $\begin{array}{l}32.9 \\
23.5 \\
\end{array}$ & 0.64 & $\begin{array}{l}0.42 \\
\mathrm{NS}\end{array}$ & $\begin{array}{c}0.63 \\
(0.20-1.98) \\
\end{array}$ \\
\hline $\begin{array}{l}\text { Frequent aero } \\
\text { plane travelling: }\end{array}$ & $\begin{array}{l}\text { No } \\
\text { Yes }\end{array}$ & $\begin{array}{c}301 \\
11\end{array}$ & $\begin{array}{c}202 \\
9\end{array}$ & $\begin{array}{l}67.1 \\
81.8\end{array}$ & $\begin{array}{c}99 \\
2\end{array}$ & $\begin{array}{c}32.90 \\
18.2\end{array}$ & 1.05 & $\begin{array}{l}0.31 \\
\mathrm{NS}\end{array}$ & $\begin{array}{c}0.45 \\
(0.10-2.14)\end{array}$ \\
\hline
\end{tabular}

$\chi^{2}$ : Chi square test. $\quad$ NS: Non-significant $(\mathrm{P}<0.05) \quad * *$ : Highly significant $(\mathrm{P}<0.01)$. OR: Odds ratio 
https://ejhm.journals.ekb.eg/

\section{DISCUSSION}

Epistaxis, bleeding from the nose, is a communal grievance. It is rarely life threatening but may cause significant concern, especially among parents of small children. Most nosebleeds are benign, self-limiting, and spontaneous, but some can be recurrent. Many uncommon causes are also noted ${ }^{(7)}$.

Epistaxis can be divided into 2 categories, anterior bleeds and posterior bleeds, on the basis of the site where the bleeding originates. The true prevalence of epistaxis is not known, because most episodes are self-limited and thus are not reported. When medical attention is needed, it is usually because of either the recurrent or severe nature of the problem. Treatment depends on the clinical picture, the experience of the treating physician, and the availability of ancillary services (8).

In this study regarding children ages and gender, $60.9 \%$ were from 10 to 12 years old and $55.8 \%$ were males. About two thirds of mothers and fathers were highly educated. Also $91.3 \%$ of fathers were working and $52.2 \%$ of mothers were housewives. Finally, $52.9 \%$ were of high social class. These results are concomitant with a study of Wahab et al. ${ }^{(9)}$ that included 34 (54\%) boys and $29(46 \%)$ girls. The patients' age ranged from 2-14 years with a mean of 3.4 years. Twenty-eight $(44.4 \%)$ patients had positive family history with similar condition. Twenty-four $(38.1 \%)$ patients had positive consanguinity and the mean age of presentation in the study of Damrose and Maddalazzo ${ }^{(10)}$ was 7.3 years, while in Brown and Berkowitz $^{(11)}$ study was 7.8 years.

This study showed that there were 211 child (67.6\%) did not have epistaxis and 101 child (32.4\%) had epistaxis so that prevalence of epistaxis among the studied group was $32.4 \%$. Study of Boohh \& McMains ${ }^{(12)}$, showed that prevalence of epistaxis was $30 \%$ of all children aged $0-5$ years, $56 \%$ of those aged $6-10$ years, and $64 \%$ of those aged 11-15 years had at least one episode of epistaxis in their lifetime.

The study showed that most frequent risk factors among the whole studied group were negative smokers, subjection to head trauma, exposure to factory gases, and complaining from chronic cough and upper respiratory tract infection $(56.7 \%, 45.8 \%, 35.6 \%, 32.7 \%$ \& $30.1 \%$ respectively). Many putative risk factors for single epistaxis events have been described by Melia \& McGarry ${ }^{(13)}$, including facial injury, physical and chemical irritation, allergic rhinitis, viral and bacterial rhinosinusitis, nasal tumors, temperature, and humidity. Our results are supported by study of Abrich et al. ${ }^{(14)}$ who reported that the most common risk factors among the studied group were rhinitis, upper respiratory tract infection and sinusitis. Although, Wahab et al. ${ }^{\left({ }^{(9)}\right.}$ found that twenty-five (39.7\%) patients had positive local nose causes of epistaxis, eighty-eight percent of these patients had bacterial rhinitis diagnosed by positive bacterial culture for nasal discharge. The most common organisms included were Streptococcus pneumonia, Haemophilus influenzae, and Moraxella catarrhalis. One-third of such patients were found to be habitual nose pickers by history taking and had positive crusting of the anterior nasal septum due to digital trauma. Another $1 / 3$ of the patients showed lodged nasal foreign body diagnosed by unilateral foul nasal discharge, inflamed mucosa, and granulation tissue around foreign body and on endoscopic examination foreign body was detected and removed. The remaining $1 / 3$ of the patients most probably represent cases of complicated previous viral rhinitis. Kasperek and Pollock ${ }^{(15)}$ said that epistaxis can also occur as a result of medication exposure and is associated with the use of antiplatelet medications for the secondary prevention of heart attack and stroke, including aspirin and clopidogrel. Iqbal and Ahmed ${ }^{(7)}$ reported that the most prevailing cause for epistaxis was trauma (50.20\%) in youngsters, which was followed by hypertension $(29.30 \%)$ in adults. According to Ali et al. ${ }^{(16)}$ more than $75 \%$ of cases of nasal trauma present with epistaxis. Trauma is involved in young age while epistaxis with hypertension was found in adult age in intrinsically disordered proteins (IDPs).

This study founded that $51.5 \%$ of the studied group had the first attack of epistaxis at 1 to 4 years ago and $58.4 \%$ had it with rate once to twice per month with $49.5 \%$ of moderate amount. Regarding seasonal variation, there was $67.3 \%$ increase in summer. In $61.4 \%$ of the cases it was associated with fatigue and lethargy, $57.4 \%$ was associated with scratching inside nose, $74.3 \%$ was idiopathic, $29.7 \%$ was after FB entrance and in $6.9 \%$ it was associated with travelling with plane. Beck et al. ${ }^{(17)}$ reported about $60 \%$ of the population experience a nosebleed at least once in their life. Precise epidemiological data on incidence are unavailable, because no epidemiological studies have been performed and only about $6 \%$ to $10 \%$ of the persons affected seek medical help. Findings of our results are in contrast with study of Davies et al. ${ }^{(18)}$, as they reported that recurrent bleeds were common, occurring in $88 \%$ of the patients in their study. With episodes occurring three times or more per week in as many as $40 \%$ of the children.

The results of our study showed that cases had head trauma were about 8 times more exposed to have epistaxis, also cases use nasal spray were 5 times more exposed to have epistaxis and cases had hemophilia was about 7 times more exposed to have epistaxis. Finally, vascular disease increased risk of epistaxis 145 times. Diamond ${ }^{(19)}$ said that epistaxis results from damage or rupture of the vasculature in the nasal cavity. While nearly $40 \%$ of cases of epistaxis have been reported to be idiopathic, there are several potential direct causes, such as trauma (nose picking, facial injury, foreign body, nasogastric tube placement, barotrauma), neoplasms and 
nasal polyps, nasal dryness, septal perforation, infection and environmental irritants such as cigarette smoke.

It was further found by Lee and Lane ${ }^{(20)}$ that epistaxis occurrence is positively associated with allergic rhinitis, chronic sinusitis, coagulopathy, hereditary hemorrhagic telangiectasia, hematologic malignancy, and hypertension. These disorders of the otolaryngological region, in particular sinusitis, are associated with an overactivation of the inflammatory system, which could result in an increased number of bleeding episodes such as epistaxis.

\section{CONCLUSIONS}

Pediatric epistaxis is a condition commonly faced by both primary health care physicians and otolaryngologists. Recurrent epistaxis can be troublesome and alarming for parents and children. The prevalence of epistaxis among the studied group was $32.4 \%$. We found that most common risk factors among the studied group were smoking, head trauma, factory gases, chronic cough and URTI. Regardless of medical specialty, first aid measures, mucosal hydration and chemical cautery form the fundamentals of managing this common condition.

\section{RECOMMENDATIONS}

Raising awareness of simple management strategies among parents and primary care physicians (PCPs) could significantly reduce the stress and severity of disease. Other researches on wider range should be applied to include more areas in the governorate and whole Egypt. Preventive measures must be taken against epistaxis to decrease incidence of bleeding. Intensify the role of family physician (GP) in controlling bleeding as he is the first contact with patient. Schools also should be interested about the diseased students and give them special care by nursing them routinely as they are more anemic than others, less in attention in class and used to absent to do to doctor or hospitals.

Study limitation: The finding presented in this study represented only a rural area (Sanafa, Mit-Ghamr), so we can't generalize these results on all the country. Uncooperation of some participants especially healthy ones so it took many time for searching other children.

Conflict of interest: No conflict of interest.

Funding: No fund.

\section{REFERENCES:}

1. Yenigun A, Akyuz S (2015): Prevalence of Allergic Rhinitis Symptoms and Positive Skin Prick Test Results in Children with Recurrent Epistaxis. British Journal of Medicine and Medical Research, 7 (3): 241-246.
2. Khan I, Kubba H (2014): Evidence-based management of paediatric epistaxis. Journal of ENT Masterclass, 7 (1): 1013.

3. Saafan M, Ibrahim W (2013): Role of bacterial biofilms in idiopathic childhood epistaxis. European Archives of Oto-Rhino-Laryngology, 270 (3): 909-914.

4. Wahab M, Fathy H, Ismail R et al. (2014): Recurrent epistaxis in children: When should we suspect coagulopathy? The Egyptian Journal of Otolaryngology, 30 (2): 106-111.

5. Al Masum S, Arsalan A, Begum D (2015): Epistaxis in Children: Aetiology, Management and Outcome. Bangladesh Journal of Child Health, 39 (2): 73 76.

6. Fahmy S, Nofal L, Shehata $\mathrm{S}$ et al. (2015): Updating indicators for scaling the socioeconomic level of families for health research. Journal of the Egyptian Public Health Association, 90 (1): 1-7.

7. Iqbal M, Ahmed W (2015): Epistaxis: Its Prevalence in IDPs of North Waziristan Agency. American Journal of Clinical and Experimental Medicine, 3 (5): 233-236.

8. Pope L, Hobbs C (2005): Epistaxis: an update on current management. Postgraduate Medical Journal, 81 (955): 309-314.

9. Wahab M, Fathy H, Ismail R et al. (2014): Recurrent epistaxis in children: When should we suspect coagulopathy? The Egyptian Journal of Otolaryngology, 30 (2): 106-111.

10. Damrose J, Maddalozzo J (2006): Pediatric epistaxis. The Laryngoscope, 116.3 (2006): 387-393.

11. Brown R, Barkowitz S (2004): Epistaxis in healthy children requiring hospital admission. Int $\mathbf{J}$ Pediatr Otolaryngol., 68: 1181-1184.

12. Booth C, McMains $K$ (2009): Pediatric epistaxis. In: Pediatric Otolaryngology for the Clinician. Mitchell and K.D. Pereira (eds.), Humana Press, a part of Springer Scienc, Business Media, LLC. Pp: 97.

13. Melia L, McGarry G (2010): Epistaxis: update on management. Curr Opin Otolaryngol Head Neck Surg., 19 (1): $30-35$.

14. Abrich V, Brozek A, Boyle T et al. (2014): Risk factors for recurrent spontaneous epistaxis. Mayo Clinic Proceedings, 89 (12): 1636-1643.

15. Kasperek Z, Pollock G (2013): Epistaxis: an overview. Emerg Med Clin North Am., 31 (2):443-54.

16. Ali S, Mumtaz S, Saeed M (2003): Epistaxis: aetiology and management. Ann King Edward Med Coll., 9: 272-4.

17. Beck R, Sorge M, Schneider A et al. (2018): Current approaches to epistaxis treatment in primary and secondary care. Deutsches Ärzteblatt International, 115 (1-2): 12-16.

18. Davies K, Batra K, Mehanna $R$ et al. (2014): Pediatric epistaxis: epidemiology, management \& impact on quality of life. International Journal of Pediatric Otorhinolaryngology, 78 (8): 1294-1297.

19. Diamond L (2014): Managing epistaxis. JAAPA., 27 (11): 35-9.

20. Lee S, Lane A (2011): Chronic Rhinosinusitis as a Multifactorial Inflammatory Disorder. Curr Infect Dis Rep., 13 (2): 159-68. 